\title{
Reimplantation of anomalous right coronary artery arising from the pulmonary trunk into the aorta is low risk and good midterm result
}

\author{
Ivanov AS ${ }^{1}$, Gureev SV*1, Glamazda SV', Ostroumov EN ${ }^{1}$ and Lugovskiy $\mathrm{MK}^{1}$ \\ ${ }^{1}$ Federal Research Centre of Transplantology and Artificial Organs named after V.I. Shumakov, Moscow, Russian Federation
}

\begin{abstract}
This report describes results of right coronary artery reimplantation with tomography, myocardial perfusion imaging and diagnostic coronary angiography evaluation before and 1 year after operation on a 9 years old boy with anomalous right coronary artery from pulmonary artery.
\end{abstract}

\section{Introduction}

The clinical profile and the natural history of Anomalous right coronary artery (RCA) from pulmonary artery (ARCAPA) are not well delineated, and the information is derived mostly from individual case reports, $[2,3,4]$. This report describes results of right coronary artery (RCA) reimplantation with tomography, myocardial perfusion imaging and diagnostic coronary angiography evaluation before and 1 year after operation on a 9 years old boy with ARCAPA.

\section{Case report}

The 9 years old boy, was hospitalized for evaluation of a heart murmur and diagnostics of coronary-pulmonary fistula. His only complaint was fatigue during the physical exercises.

Spiral contrast computed tomography: Left coronary artery (LCA) located typically. LCA trunk diameter-7 $\mathrm{mm}$. Anterior descending artery diameter- $6 \mathrm{~mm}$. Left circumflex artery diameter- $5 \mathrm{~mm}$, both located typically. RCA originates from the trunk of pulmonary artery (Figure 1B). Diagnostic coronary angiography confirmed these findings and demonstrated that RCA was dilated along all its length and the retrograde flow of contrasted blood from LCA filled RCA through the collateral system and went further into pulmonary artery (Figure 1A). Myocardial perfusion imaging with ECG-gated single-photon emission computed tomography (SPECT) with $170 \mathrm{mBk}$ MIBI-Tc99m: left ventricular myocardium with the areas of mild hypoperfusion in the anterior septal zone (Figure 2C).

\section{Operation}

RCA trunk was decided to fulfil translocation of the RCA mouth from the pulmonary artery to ascending aorta without extracorporeal circulation on the beating heart. RCA mouth has been ligated, cut, and subsequently RCA was re-implanted into aorta. Antegrade flow through it was documented. The result of operation see on Figure 2A.

\section{Result}

Areas of Left Ventricular anterior and septal walls hypoperfusion on myocardial perfusion imaging disappeared after RCA reimplantation (Figure 2 D).

\section{One year after the operation}

According to his mother, he became more active than before the operation. Diagnostic coronary angiography: contrasted blood flow through the arteries was normal. Right coronary artery originates from the aorta, its proximal part was dilated to $11 \mathrm{~mm}$ (from $7 \mathrm{~mm}$ ), there was an area of $4 \mathrm{~mm}$ stenosis on the border of the medium and proximal parts of the artery. The large proximal branch RCA was obliterated. (Indicated by arrow) (Figure 2 B).

\section{Discussion}

ARCAPA is a very rare congenital anomaly first described in 1885 by Brooks, [1]. This condition, when not combined with other congenital heart defects, is often asymptomatic and is found incidentally. However, there are some patients with ARCAPA, who suffer from exertion angina, and the cases of sudden death were reported as well [5]. When RCA originates from the pulmonary artery, steal syndrome may cause exercise-induced myocardial ischemia. This may be the mechanism of sudden death, and if it is true, then surgical correction appears warranted.

In our case this approach provided for the clinical improvement, normalization of myocardial perfusion and diastolic function of both ventriculars and reduction of LV asynchrony and increase in EF of RV from 20 to $34 \%$ in one year.

In one year after RCA translocation the dilatation of its proximal part with its subsequent stenosis was detected. Similar situation was described by Veselka J, [4]. We assume that this dilatation was due to the redirection of the blood flow through RCA, and the pressure increase within RCA, which has been previously adapted to the low

Correspondence to: Sergey V Gureev, Federal reseach centre of transplantology named after Shumakov Moscow, Moscow Russian Federation, Tel: +07 916 6910996; E-mail: svgdoctor@gmail.com

Key words: anomalous right coronary artery, reimplantation

Received: April 22, 2017; Accepted: May 29, 2017; Published: May 31, 2017 


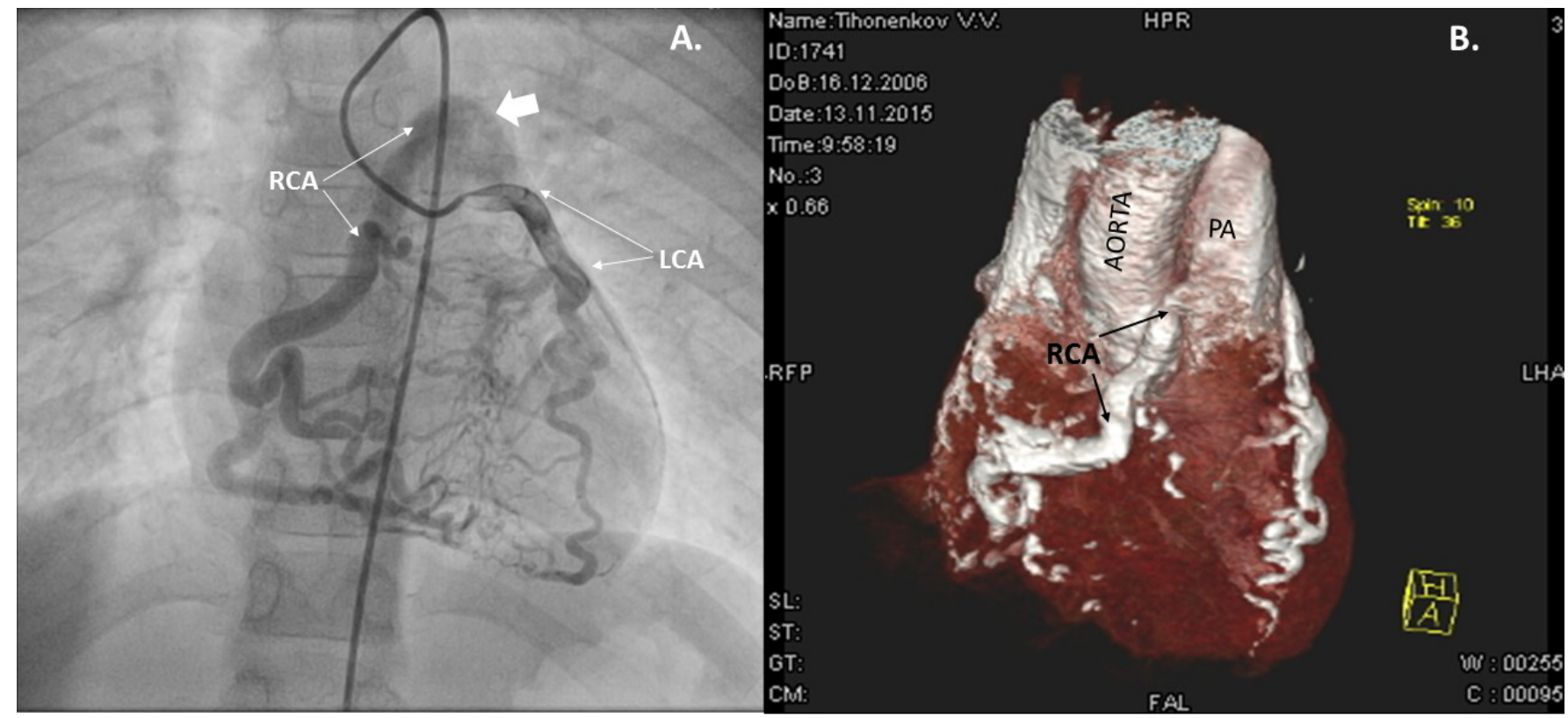

Figure 1. A) Coronary angiography before operation, went into pulmonary artery indicated by arrow. B) Computed tomography before operation. RCA-right coronary artery, LCA-left coronary artery, $\mathrm{PA}$ - pulmonary artery.

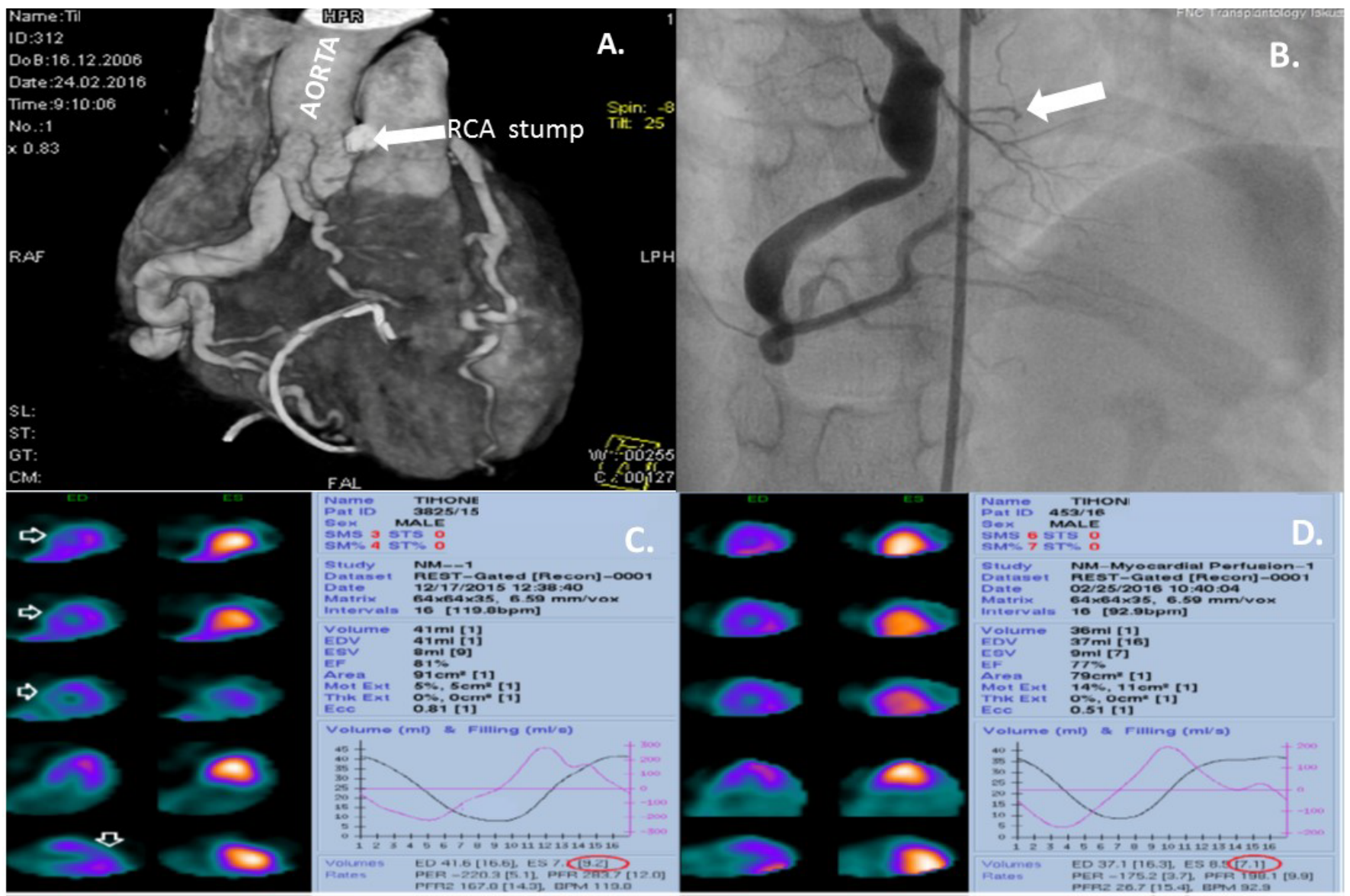

Figure 2. A) Computed tomography 5 days after RCA re-implantation. B) Coronary angiography of RCA 1 year after RCA reimplantation; C/D - Myocardial perfusion imaging. C) (before operation) - left ventricular myocardium with the areas of mild hypoperfusion in the anterior septal zone. D) (after operation) - Areas of LV anterior and septal walls hypoperfusion on myocardial perfusion imaging with ECG-gated single-photon emission computed tomography disappeared after RCA reimplantation. 
pressure. Vice versa, - the LCA diameter has decreased in comparison with preoperative data. The decsrease of LCA diameter was probably due to the improvement of capillary blood flow and the decrease in vascular resistance.

\section{Conclusion}

Although most of the patients with ARCAPA remain asymptomatic, reimplantation of the RCA into the aorta may be recommended to prevent myocardial hypoperfusion and its consequences.

\section{References}

1. Brooks HSJ (1885) Two Cases of an Abnormal Coronary Artery of the Heart Arising from the Pulmonary Artery. J Anat Physiol 20(Pt 1): 26-29. [Crossref]
2. Williams IA, Gersony WM, Hellenbrand WE (2006) Anomalous right coronary artery arising from the pulmonary artery: a report of 7 cases and a review of the literature. $A m$ Heart $J$ 152:1004. [Crossref]

3. Refatllari A, Likaj E, Dumani S, Hasimi E, and Goda A (2015) Surgical Treatment of Anomalous Origin of Right Coronary Artery in a Patient with Mitral Stenosis. Open Access Maced J Med Sci 15;4(1):131-4. [Crossref]

4. Veselka J, Widimsky' P, Kautzner J (2003) Reimplantation of anomalous right coronary artery arising from the pulmonary trunk leading to normal coronary flow reserve late after surgery. Ann Thorac Surg 76: 1287-1289. [Crossref]

5. Click RL, Holmes DR Jr, Vlietstra RE, Kosinski AS, Kronmal RA (1989) Anomalous coronary arteries: location, degree of atherosclerosis and effect on survival: a report from the Coronary Artery Surgery Study. J Am Coll Cardiol 13:531-537. [Crossref]

Copyright: $\odot 2017$ Ivanov AS. This is an open-access article distributed under the terms of the Creative Commons Attribution License, which permits unrestricted use, distribution, and reproduction in any medium, provided the original author and source are credited. 